\title{
UV/ozone surface treatment increases hydrophilicity and enhances functionality of SU-8 photoresist polymer
}

\author{
Aleksandra Delplanque ${ }^{a}$, Etienne Henry ${ }^{a}$, Joseph Lautru ${ }^{b}$, Hervé Leh $^{a}$, \\ Malcolm Buckle ${ }^{\mathrm{a}}$, Claude Nogues ${ }^{\mathrm{a}, *}$ \\ a Laboratory of Applied Biology and Pharmacology, ENS de Cachan, CNRS; 61 Avenue du President Wilson, 94230 Cachan, France \\ ${ }^{\mathrm{b}}$ The Alembert Institute, ENS de Cachan; 61 Avenue du President Wilson, 94230 Cachan, France
}

\section{A R T I C L E I N F O}

\section{Article history:}

Received 21 January 2014

Received in revised form 8 May 2014

Accepted 9 June 2014

Available online 7 July 2014

\section{Keywords:}

SU-8 polymer

UV/ozone

Surface (bio) functionalization

Monolayer

\begin{abstract}
A B S T R A C T
SU-8 photoresist polymer is widely used in the fabrication of microdevices. However, for biological applications, the problem of efficiently modifying SU-8 surfaces without perturbing roughness has not been successfully resolved. We present UV/ozone (UVO) surface pre-treatment as an effective method to increase the hydrophilicity of SU-8 films without affecting surface roughness, thus improving specific covalent binding of bio-molecules. We demonstrate that $30 \mathrm{~s}$ UVO treatment suffices to create carboxyl groups at the surface that can then be used for high density binding of molecules via amide bond formation. We further demonstrate that a two-step surface modification where the surface is first protected with an ethylene glycol monolayer leads to an increase in binding specificity. Finally, to illustrate the controlled binding and accessibility of immobilized molecules, we show three cycles of reversible interactions between anti-tamra antibody and tamra-cadaverine immobilized on the surface of SU-8.
\end{abstract}

\section{Introduction}

SU-8 is a negative photosensitive polymer with a low Young's modulus and excellent chemical and thermal stability, widely used in the manufacture of extremely robust microstructures $[1,2]$. The optical properties of SU-8, including transparency above $360 \mathrm{~nm}$, coupled with its relatively low refractive index, make it an ideal material for optical waveguides and the development of label-free biosensors $[3,4]$.

However, the surface of SU-8 is highly hydrophobic, thus incompatible with many applications in biotechnology [5-7]. Surface modifications of SU-8 to enhance its wettability and to allow controlled immobilization of (bio)molecules of interest have been developed [8-11]. Modifications essentially addressed the means of activating SU-8 surfaces using wet or dry pre-treatment methods. Wet pre-treatment methods use a cross-linker that reacts with hydroxyl and/or carboxyl groups generated by acid and/or alkali treatment [7-9]. Dry pre-treatment methods use oxygen or ammonia plasma [12] or pyrolytic dissociation of ammonia [10]. In both cases surface roughness increases significantly; this is detrimental for liquid distribution in a static fluid microdevice and results in an unsteady secondary flow in a flowing microchannel $[13,14]$.

\footnotetext{
* Corresponding author. Tel.: +33014740 7687

E-mail address: claude.nogues@lbpa.ens-cachan.fr (C. Nogues).
}

In addition, surface roughness induces optical loss in waveguides due to scattering on sidewalls [15] and promotes non-specific adsorption of (bio)molecules to the surface [16].

In this paper we present UV/ozone (UVO) pre-treatment as a straightforward method to activate SU-8 surfaces [17-19] and control chemisorption of molecules [3,4]. Because UVO leads to a milder surface oxidation than argon or oxygen plasma treatment, the SU-8 surface roughness is not affected by UVO exposition. Using water contact angle measurements (wCA), FTIR spectroscopy and AFM, we characterized treated SU-8 films and, taking advantage of the fluorescence properties of tamracadaverine, we optimized experimental conditions to favor specific chemisorption of biomolecules on the UVO activated SU-8 surface.

To reinforce the specificity of biomolecule adsorption on the UVO activated SU-8 surface we compared one-step direct adsorption of biomolecules with a two-step adsorption process in which biomolecules were immobilized following the deposition of an anti-fouling layer composed of oligomers of ethylene glycol [20-22].

Finally, to illustrate biosensing applications, we followed the decrease of the fluorescence intensity upon association of antitamra with immobilized tamra-cadaverine. We demonstrate not only that the surface modification allows controlled immobilization of biomolecules but also does not impact on their functional integrity. 


\section{Materials and methods}

\subsection{Substrates and chemical reagents}

Experiments were performed with double distilled water all buffers were filtered before use with a low protein binding non-pyrogenic $0.8 / 0.2 \mu \mathrm{m}$ filter. Silicon wafers used as a substrate were purchased from ACM-PVD (France). SU-8 2002 was purchased from Micro-Chem (USA). N-[3-Dimethylaminopropyl]-N'-ethylcarbodiimide hydrochloride (EDC) and $\mathrm{N}$ Hydroxysuccinimide (NHS) were purchased from Sigma-Aldrich. Carboxy-amine $n$-ethylene glycol reagents $\left(\mathrm{CA}(\mathrm{EG})_{8}\right.$ and $\left.\mathrm{CA}(\mathrm{EG})_{24}\right)$ were purchased from Thermo Scientific. Tetramethylrhodamine (tamra) cadaverine and anti-tetramethylrhodamine rabbit IgG fraction (anti-tamra) were purchased from Invitrogen - Molecular Probes.

\subsection{Fabrication of the SU-8 photoresist film}

2-inch silicon wafers were cleaned for $10 \mathrm{~min}$ in the UVOCleaner (model 42-220, Jelight, USA). Microscope glass slides were cleaned with ethanol and acetone followed by cleaning in the UVO-Cleaner for $10 \mathrm{~min}$. 2-3 $\mu \mathrm{m}$ thick SU-8 films were obtained, following the method developed by Camille Delezoïde [3,4,23]. It consists in spin coating of SU-8 2002 at $500 \mathrm{rpm}$ for $7 \mathrm{~s}$ with an acceleration of $100 \mathrm{rpm} / \mathrm{s}$; followed by $3000 \mathrm{rpm}$ for $30 \mathrm{~s}$ with an acceleration of $300 \mathrm{rpm} / \mathrm{s}$ using Spin-Coater RC8, Suss MicroTec. The films were softbaked on a hotplate for $1 \mathrm{~min}$ at $65^{\circ} \mathrm{C}$, followed by 2 min at $95^{\circ} \mathrm{C}$ for a silicon wafer and at $65^{\circ} \mathrm{C}$ for $6 \mathrm{~min}$ for a glass slide. Samples were flood-exposed in UV light for $15 \mathrm{~s}$, using the UV-Aligner MJB4, Suss MicroTec. Post-exposure baking was performed on a hotplate for $1 \mathrm{~min}$ at $65^{\circ} \mathrm{C}$, followed by $3 \mathrm{~min}$ at $95^{\circ} \mathrm{C}$ for a silicon wafer and $6 \mathrm{~min}$ at $95^{\circ} \mathrm{C}$ for a glass slide. Samples were then hardbaked under three different conditions: at $90^{\circ} \mathrm{C}$ for $5 \mathrm{~h}$, at $120^{\circ} \mathrm{C}$ for $3 \mathrm{~h}$ or at $180^{\circ} \mathrm{C}$ for $2 \mathrm{~h}$. The thickness of the films was controlled using Dektak 150 Surface Profiler, Veeco. Prepared surfaces were stored under nitrogen or used directly in the following experiments.

\subsection{UV/ozone (UVO) treatment}

Prepared surfaces were exposed to the UVO using the UVOCleaner (low pressure mercury vapor grid lamp: $28,000 \mu \mathrm{W} / \mathrm{cm}^{2}$ at $254 \mathrm{~nm}$; model 42-220, Jelight, USA). Exposure of the samples to UVO lasted from $15 \mathrm{~s}$ till $20 \mathrm{~min}$.

\subsection{Fourier transform infrared spectroscopy (FTIR)}

The surfaces before and after $30 \mathrm{~s}$ and 5 min of UVO treatment were analyzed in FT-IR spectrometer with resolution of $8.00 \mathrm{~cm}^{-1}$, 1000 scans (apparatus Nexus - Thermo Electron Corporation, USA). A silicon wafer was used as a background. All measurements were carried out in adsorption mode.

\subsection{Atomic force microscopy (AFM)}

Surface topography and roughness before and after $5 \mathrm{~min}$ of UVO treatment were measured using microcantilever tips OMCL-AC240TS-R3 (Olympus, Japan) in Veeco nanoscope V. Measurements were performed in tapping mode with a scan rate of $1 \mathrm{~Hz}$ and scan sizes of 1 and $5 \mu \mathrm{m}$. Images were taken at minimum three randomly selected areas. The software option 'Section' was used to calculate a root mean surface roughness (spectral RMS amplitude).

\subsection{Water contact angle measurements (wCA)}

wCA were measured on SU-8 films before and after UVO treatment. Measurements were carried out with Digidrop MCAT Analyzer (GBX, France). All measurements were taken at room temperature. $0.5 \mu \mathrm{l}$ water droplets were dispensed from a needle and the wCA was calculated on captured images, $500 \mathrm{~ms}$ after placing a drop on the surface. Each given wCA was the average value of five consecutive contact angle measurements.

\subsection{Binding of tamra-cadaverine}

The surfaces before and after 15 and $30 \mathrm{~s}, 1,2,5$ and $15 \mathrm{~min}$ of UVO treatment were immersed in a diluted solution of EDC/NHS (ratio 1:1, EDC: $0.4 \mathrm{M}$ N-[3-Dimethylaminopropyl]-N'-ethylcarbodiimide hydrochloride; NHS: $0.1 \mathrm{M}$ $\mathrm{N}$-Hydroxysuccinimide) [24] for 30-60 min. After rinsing with double distilled water, samples were incubated $1 \mathrm{~h}$ either directly with $10 \mu \mathrm{M}$ tamra-cadaverine in $10 \mathrm{mM}$ NaOAc buffer pH 5.2 or with $0.1 \mathrm{mM} \mathrm{CA}(E G)_{8}$ or $\mathrm{CA}(\mathrm{EG})_{24}$ diluted in water. For pegylated SU-8 surfaces, samples were again immersed in EDC/NHS for $1 \mathrm{~h}$ and then rinsed with a buffer or sonicated twice for $2 \mathrm{~min}$. Dried surfaces were analyzed in Typhoon scanner with an emission filter of $580 \mathrm{~nm}$. The density of tamra-cadaverine on the SU-8 surface was deduced from a calibration curve: using a Typhoon imager, the fluorescence intensity of $1 \mu \mathrm{l}$ of an increasing concentration of tamra-cadaverine solution was measured to obtain a linear calibration curve. The concentration range used for the calibration curve was chosen according to the contrast obtained after scanning all samples with a Typhoon scanner so the calibration curves and the number of moles per unit area for each sample was within the same intensity range.

\subsection{Tamra-cadaverine/anti-tamra interactions}

Pretreated SU-8 thin films were used to spot $10 \mu \mathrm{M}$ tamracadaverine in $10 \mathrm{mM} \mathrm{NaOAc}$ buffer pH 5.2 with $1 \% \mathrm{v} / \mathrm{v}$ glycerol. Spots with the dimension of around $300 \mu \mathrm{m}$ were deposited using a Vikalex Spotter (Vikalex Technologies, France). $10 \mu \mathrm{g} / \mathrm{ml}$ of antitamra diluted in PBS buffer was incubated on the tamra-cadaverine functionalized surface for $1 \mathrm{~h}$ after which the surface was rinsed with $10 \mathrm{mM}$ glycine- $\mathrm{HCl} \mathrm{pH} 2.0$ to remove antibodies retained on the surface. All reactions were performed under liquid on the protected SU-8 surface; microfluidic flowcells were prepared on the glass slides using Sticky Slides $\mathrm{VI}^{0.4}$ (ibidi, Germany). Three cycles were carried out for the reaction between immobilized tamra-cadaverine molecules and anti-tamra immobilized on the surface in a microfluidic flowcell, followed by surface regeneration with a glycine solution. Fluorescence images were recorded using an inverted epifluorescence microscope (Leica MicroSystems) equipped with a $4 \times$ objective and fluorescence filters suitable for tamra (excitation bandpass $510-560 \mathrm{~nm}$, emission bandpass $590-680 \mathrm{~nm}$ ). Average fluorescence intensity was calculated using ImageJ Software (NIH) after subtracting a background for each spot.

\section{Results and discussion}

\subsection{Characterization of SU-8 surface after UV/ozone (UVO) treatment}

In order to access a range of cross-linking densities on the SU-8 surface [25-27], we studied three hardbaking conditions following UVO exposure: $90^{\circ} \mathrm{C}$ for $5 \mathrm{~h}, 120^{\circ} \mathrm{C}$ for $3 \mathrm{~h}$ and $180^{\circ} \mathrm{C}$ for $2 \mathrm{~h}$. The degree of SU-8 reticulation, and thus the amount of reactive epoxy groups present on the surface, is largely conditioned by the hardbaking step $[25,26,28]$. The impact of UVO exposure time on 


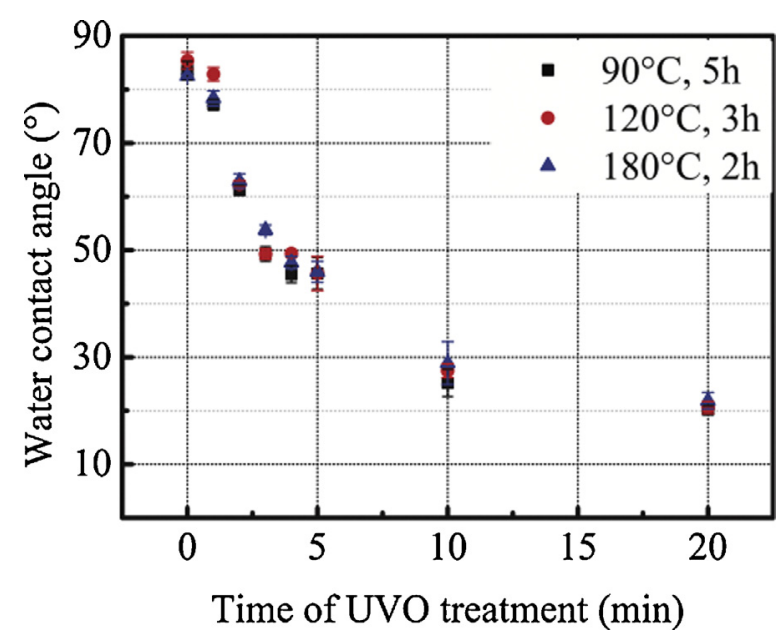

Fig. 1. Variation of the wCA with increasing UVO exposure time of SU-8 thin film prepared with three different hardbaking conditions. Each data point corresponds to the average of five wCA measurements.

SU-8 surfaces prepared under these three different conditions was followed using wCA, AFM and FT-IR spectroscopy.

wCA allowed characterization of the evolution of the SU-8 surface energy for each UVO exposure time. As shown in Fig. 1, starting from a wCA of $83.5 \pm 1.5$, independently of the hardbaking conditions and in agreement with published data $[7,9,28]$, the wCA decreased below $30^{\circ}$ after 10 min of UVO exposure and reached a plateau around $22^{\circ}$ after $20 \mathrm{~min}$ of UVO treatment.

The increase of surface energy of the SU-8 surface after UVO treatment might be explained by the creation of polar groups at the SU-8 surface upon oxidation [28]. Using a surface profiler we demonstrated that the thickness of the SU-8 layer was not affected after 20 min of UVO exposure (data not shown). In addition, it has been demonstrated that the optical properties of an SU-8 waveguide were not altered by such UVO treatment $[3,4]$.

wCA measurements were extremely reproducible and no significant difference between the left and the right wCA $\left( \pm 0.3^{\circ}\right)$ could be measured, indicating that the UVO treatment did not affect the SU-8 surface roughness.
To confirm this last point, both peak-to-valley and root-meansquare (RMS) roughness were measured by AFM on the three SU-8 samples before and after UVO treatment. Fig. 2 shows AFM images and the corresponding height profiles with the RMS roughness analysis of SU- 8 thin film hardbaked at $120^{\circ} \mathrm{C}$ for $3 \mathrm{~h}$ before and after $5 \mathrm{~min}$ of UVO treatment. The SU- 8 surface before UVO is homogenous, with a peak-to-valley roughness of $1.7 \mathrm{~nm}$ and a RMS roughness of $0.121 \mathrm{~nm}$. After 5 min under UVO, the surface remains homogenous with a peak-to-valley roughness of $1.9 \mathrm{~nm}$ and a RMS roughness of $0.144 \mathrm{~nm}$. In addition, postexposure hardbaking conditions had no significant influence on the SU-8 surface roughness before and after UVO treatment time (Fig. 2c).

We analyzed SU-8 thin layers prepared using FT-IR spectroscopy (Fig. 3). The most relevant signals for the SU-8 photoresist are: 862 and $913 \mathrm{~cm}^{-1}$ corresponding to the epoxy ring [29]; between 1030 and $1295 \mathrm{~cm}^{-1}$ for C-O and C-O-C vibrations; between 1502 and $1604 \mathrm{~cm}^{-1}$ corresponding to phenyl groups and at around $1700 \mathrm{~cm}^{-1}$ a wide peak corresponding to the $\mathrm{C}=\mathrm{O}$ of a carbonyl group [10,33-34].

While the intensity of peaks corresponding to the long molecular chains of SU-8 polymer was not affected by UVO treatment, significant variations were observed for peaks corresponding to epoxy groups and to carboxyl groups [28]. As the UVO treatment time increased, the intensity of the signal from the epoxy groups decreased concomitantly with an increase in peak intensity corresponding to carboxyl groups.

Most of the epoxy groups within the thin SU-8 layer cross-linked during the post-exposure baking step [27,28], we thus deduced that the small peak intensities of the epoxy groups corresponded to epoxy groups present on the surface of the SU-8 film. In addition, the increase in carboxyl group peak intensity after 5 min of UVO treatment was larger than the decrease in the intensity of the epoxy groups peaks, indicating that oxidation of SU-8 was reaching the bulk of the SU-8 layer after relatively long UVO exposure time ( $5 \mathrm{~min}$ ). Therefore, activation of the SU-8 surface with $30 \mathrm{~s}$ UVO exposure time should allow efficient (bio)molecule immobilization.

Of particular interest was that, although there was the presence of carboxyl groups and lower peak intensities corresponding a)
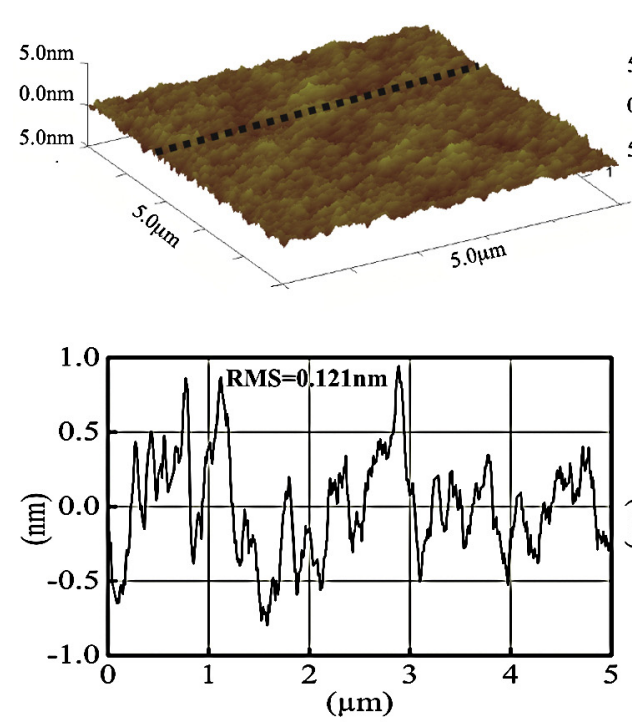

b)
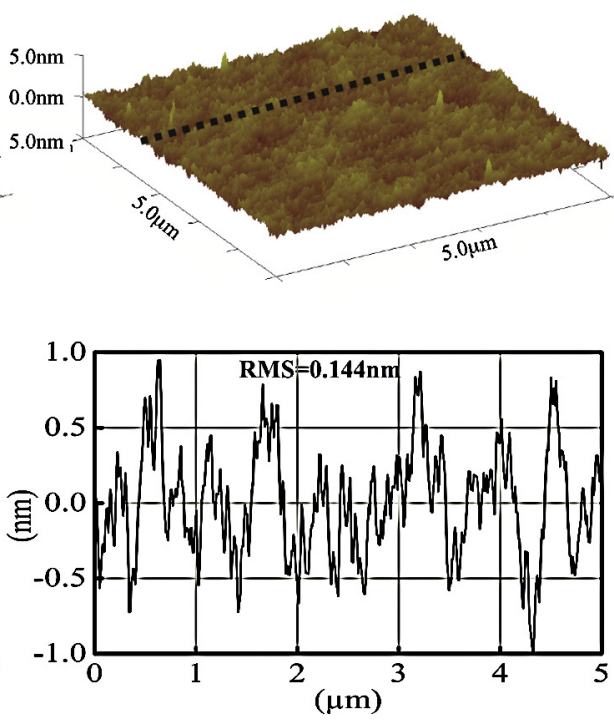

c)

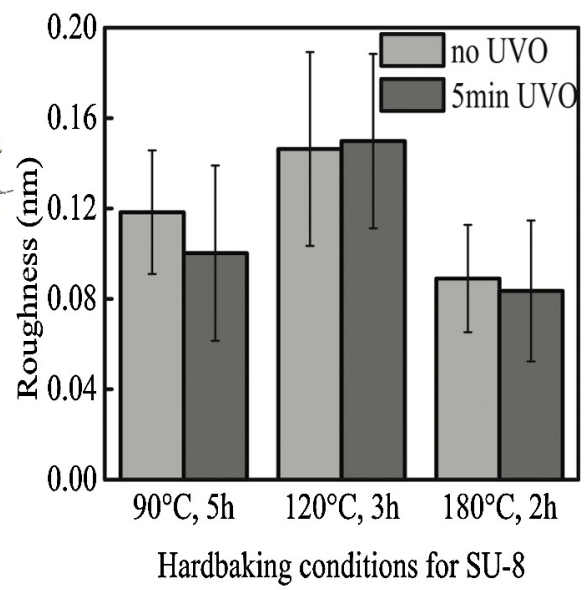

Hardbaking conditions for SU-8

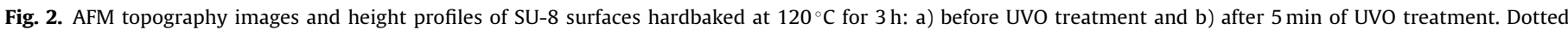

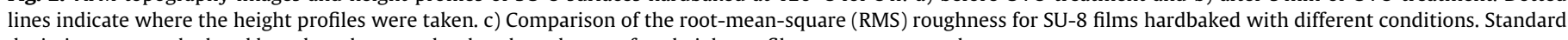
deviations were calculated based on three randomly selected areas; four height profiles per area were taken. 


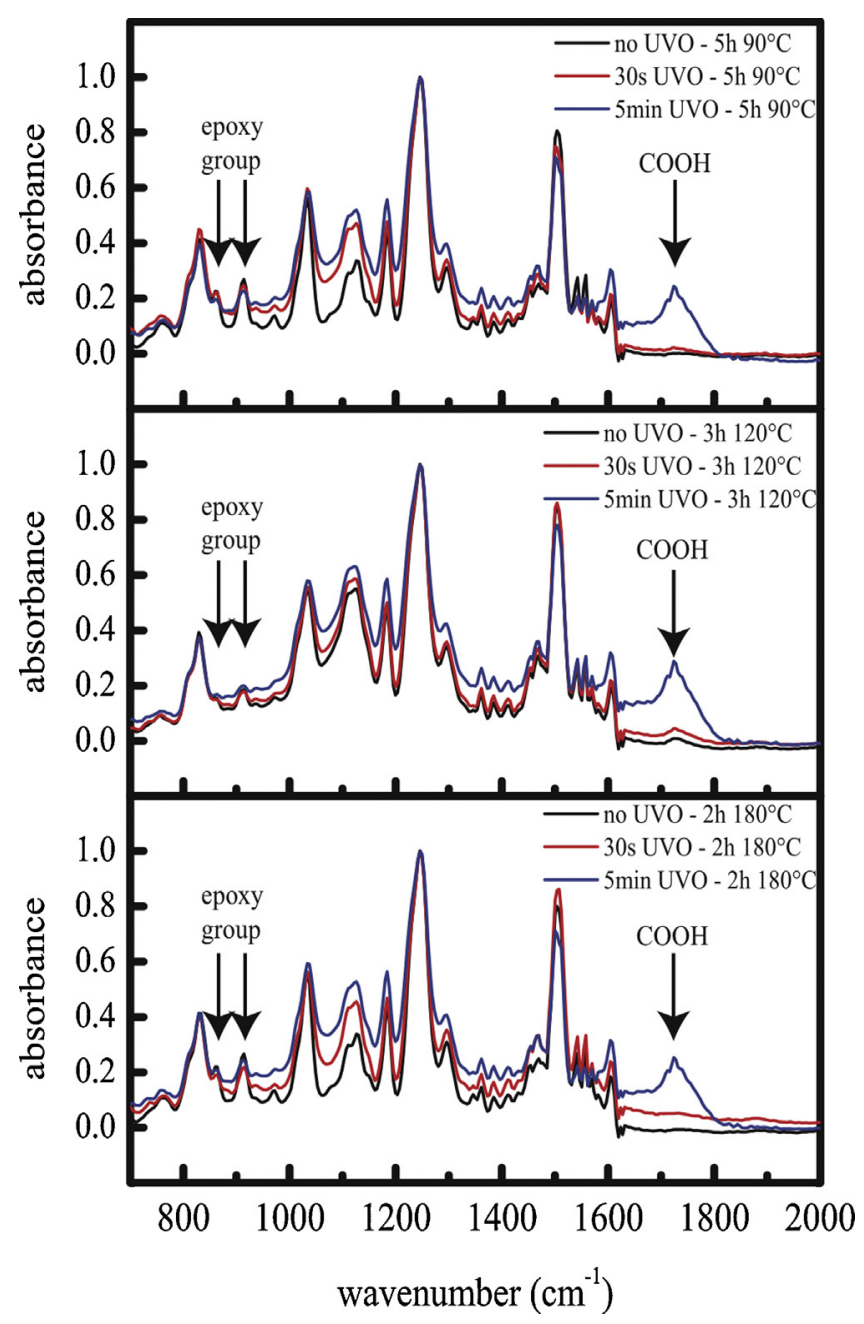

Fig. 3. FT-IR spectra of SU-8 film prepared with three hardbaking conditions indicated in the legend before and after $30 \mathrm{~s}$ and $5 \mathrm{~min}$ of UVO treatment. Peaks of epoxy (862 and $913 \mathrm{~cm}^{-1}$ ) and carboxyl groups (wide peak around $1700 \mathrm{~cm}^{-1}$ ) are indicated with arrows. All spectra were normalized to the peak at $1246 \mathrm{~cm}^{-1}$.

to epoxy groups on the SU-8 film hardbaked at $120^{\circ} \mathrm{C}$ for $3 \mathrm{~h}$ before UVO treatment, yet no carboxyl groups were detected on the SU8 film prepared under the other two hardbaking conditions. The presence of carboxylic groups on the SU-8 surface could be due to oxidation of epoxy groups at the surface and/or to the presence of some residual solvent from the thin SU-8 film [25]. This observation is, in both hypotheses, coupled with a lower peak intensity of the epoxy group on the surface of SU- 8 hardbaked at $120^{\circ} \mathrm{C}$ for $3 \mathrm{~h}$ than with the other hard baking conditions.

FT-IR revealed that changes in the oxidation state of the SU-8 layer occurred both at the surface and in the bulk of the SU-8 film for long UVO exposure time (up to $5 \mathrm{~min}$ ). While the amount of reactive epoxy groups on the SU- 8 surface is defined by the degree of reticulation of the polymer upon the post exposure hardbaking, the surface density of carboxyl groups can be potentially controlled by short UVO exposure time ( $30 \mathrm{~s}$ ). Consequently, the exposure time of SU-8 microstructure to UVO should allow modulation of the surface density of subsequent (bio)molecules following chemisorption.

\subsection{Covalent binding of tamra-cadaverine to the surface}

We used chemisorption of tamra-cadaverine to activated SU8 surfaces through specific reactions with carboxyl groups via an EDC/NHS-mediated reaction to form amide bonds at the interface
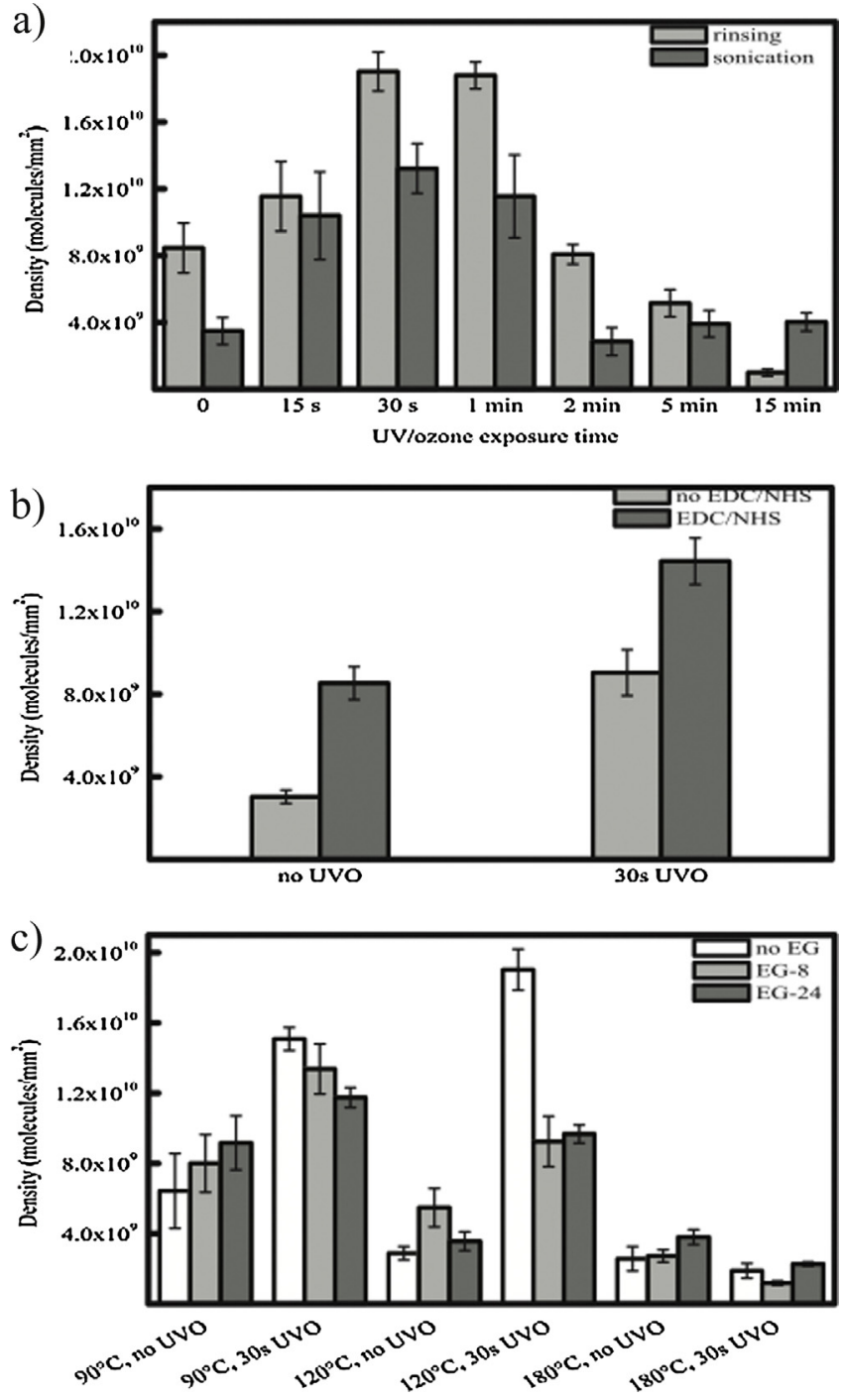

Fig. 4. Density of tamra-cadaverine molecules per $\mathrm{mm}^{2}$ immobilized on the SU8 surfaces, a) depending on UVO exposure time and rinsing method; SU- 8 thin film hardbaked at $120^{\circ} \mathrm{C}$ for $3 \mathrm{~h}$, rinsed twice with $10 \mathrm{mM} \mathrm{NaOAc}$ buffer pH 5.2 or sonicated twice for $2 \mathrm{~min}$; b) dependance on the EDC/NHS pre-incubation step c) depending on the surface pegylation method; SU-8 hardbaked in three different conditions and with or without activation by 30 -s UVO treatment. Standard deviations were calculated based on six independent measurements.

and provide a useful model to quantify the amount of material retained on the surface by measuring the intensity of tamra fluorescence when excited at $580 \mathrm{~nm}$.

Following SU-8 substrate preparation [3,4], surfaces were exposed to UVO for times ranging from 0 to $15 \mathrm{~min}$ and sequentially incubated with a solution of EDC/NHS for $60 \mathrm{~min}$, thoroughly rinsed with distilled water and incubated with $10 \mu \mathrm{M}$ solution of tamra-cadaverine for $60 \mathrm{~min}$ (see Section 2.7). Samples were then dipped in $10 \mathrm{ml}$ of buffer ( $10 \mathrm{mM} \mathrm{NaOAc}, \mathrm{pH} 5.2)$ and either agitated for $2 \times 20 \mathrm{~min}$ or sonicated twice for $2 \mathrm{~min}$ in the same buffer. The influence of UVO treatment time on the density of immobilized tamra-cadaverine molecules on the SU-8 sample hardbaked at $120^{\circ} \mathrm{C}$ for $3 \mathrm{~h}$ is shown in Fig. 4 a. For the SU- 8 not exposed to UVO treatment, the density of surface retained tamra-cadaverine was not negligible. In addition, the surface molecular density after sonication was significantly lower than for samples rinsed thoroughly and agitated in a large volume of buffer, indicating that remaining molecules were covalently attached to the SU-8 surface. It follows that the presence of residual reactive epoxy and 
carboxyl groups at the interface, highlighted by FT-IR (Fig. 3), was in itself sufficient to allow some specific binding. Second, the molecular density on the surface increased for UVO treatment lasting less than $1 \mathrm{~min}$ and decreased significantly with longer exposure. In all cases, sonication of samples, removing most non-covalently bound molecules strengthens the observation that the exposure of SU-8 surfaces between $30 \mathrm{~s}$ and $1 \mathrm{~min}$ of UVO treatment created the highest density of reactive carboxyl groups at the interface. Longer UVO exposure time most likely oxidizes SU-8 surfaces forming unreactive chemical groups, leading to lower densities of bound molecules. To conclude, the highest density of immobilized molecules $\left(1.9 \times 10^{10}\right.$ molecules $\left./ \mathrm{mm}^{2}\right)$ was obtained after $30 \mathrm{~s}$ or $1 \mathrm{~min}$ of UVO exposure.

To demonstrate the specific adsorption of tamra-cadaverine on the UVO activated surface, we characterized the molecular density on the SU-8 surface avoiding the EDC/NHS pre-incubation step (Fig. 4b). The density of tamra-cadaverine increased significantly when EDC/NHS was applied on SU-8 thin layer both with and without UVO surface treatment, confirming the specific adsorption of tamra-cadaverine on the SU-8 surface. The tamra-cadaverine density on the surface without UVO was three times higher after pre-incubation with EDC/NHS than without $\mathrm{EDC} / \mathrm{NHS}$ pre-treatment, confirming the presence of $\mathrm{COOH}$ groups on the surface hardbaked at $120^{\circ} \mathrm{C}$ for $3 \mathrm{~h}$ and the low level of non-specific interactions (corresponding to $1 / 3$ of the specifically bound tamra-cadaverine). After 30 s UVO treatment the level of non-specific binding of tamra-cadaverine increased three fold (when no EDC/NHS was applied to the surface) relative to the SU-8 surface without UVO treatment. Finally the surface density was $1.4 \times 10^{10}$ molecules $/ \mathrm{mm}^{2}$ while the non-specific interaction was $9.0 \times 10^{9}$ molecules $/ \mathrm{mm}^{2}$ when both UVO and EDC/NHS were applied to the SU-8 surface prior to the tamra-cadaverine deposition. It is important to note that the amount of non-specific interactions of tamra-cadaverine with the SU-8 surface after UVO treatment and with EDC/NHS pretreatment is expected to be significantly lower than the non-specific interactions when no EDC/NHS is employed. Indeed EDC/NHS pretreatment activates the $\mathrm{COOH}$ groups so the specific interaction (formation of an amide bond) is largely favored over the non-specific interactions, as demonstrated on the sample with no UVO treatment.

Although rinsing by sonication was shown to efficiently remove non-covalently bound molecules, it may pose a problem for biological applications through denaturation of bio molecules. Thus, to decrease non specific interactions of tamra-cadaverine and subsequent anti-tamra adsorption with the SU-8 surface, we studied the effect of pre-functionalization with carboxyl-amine $n$-ethylene glycol $\left(\mathrm{CA}(\mathrm{EG})_{8}\right.$ and $\left.\mathrm{CA}(\mathrm{EG})_{24}\right)$ [20-22].

The influence of the EG chain-length and short UVO treatment on the density of tamra-cadaverine for each SU-8 hardbaking condition is presented in Fig. 4c. We confirmed that short UVO treatment significantly increased tamra-cadaverine density on the bare surface for the three-hardbaking conditions (Fig. 4c, no EG). Furthermore, we clearly observed that hardbaking conditions influenced molecular density. Thus, in agreement with data presented above and for samples with no UVO treatment and no EG, the tamra-cadaverine surface density was the highest after hardbaking at $120^{\circ} \mathrm{C}$ for $3 \mathrm{~h}$. Although preimmobilization of $\mathrm{CA}(\mathrm{EG})_{8}$ or $\mathrm{CA}(\mathrm{EG})_{24}$ did not significantly influence tamra-cadaverine surface density for SU-8 film hardbaked at $90^{\circ} \mathrm{C}$ for $5 \mathrm{~h}$ and $180^{\circ} \mathrm{C}$ for $2 \mathrm{~h}$, we observed a lower tamra-cadaverine surface density for samples pre-functionalized with $\mathrm{CA}(\mathrm{EG})_{8}$ or $\mathrm{CA}(\mathrm{EG})_{24}$ and hardbaked at $120^{\circ} \mathrm{C}$ for $3 \mathrm{~h}$. When compared to the tamra-cadaverine surface density after sonication (Fig. 4a), we noticed that the amplitude of the drop in molecular density was similar, indicating that pre-treatment of the surface with $\mathrm{CA}(\mathrm{EG})_{8}$ or $\mathrm{CA}(\mathrm{EG})_{24}$ produced an efficient anti-fouling layer. Tamra-cadaverine surface density was lower following immobilization on pre-functionalized SU-8 surfaces than on a bare SU-8 film treated with sonication $\left(9.5 \times 10^{9}\right.$ compared to $1.3 \times 10^{10}$ molecules $/ \mathrm{mm}^{2}$ respectively) indicating that the final amount of reactive carboxyl groups was lower. The EG chain-length had no influence on the tamra-cadaverine surface density.

The SU- 8 surface hardbaked at $120^{\circ} \mathrm{C}$ for $3 \mathrm{~h}$ then activated by 30 s UVO treatment was adopted as a standard protocol for preparing SU-8 surfaces because it resulted in homogeneous samples with fewer disparities in the density of immobilized molecules (Fig. 4c).

To demonstrate the relevance of the surface modification method for bio-sensing applications, we analyzed fluorescence quenching following the interaction of anti-tamra with immobilized tamra-cadaverine.

Fluorescent microscope images at different times following injection of anti-tamra across the pre-treated SU-8 surface containing spots of immobilized tamra-cadaverine are shown in Fig. 5a. The relatively small variations in fluorescence intensity observed during the three cycles of interaction/regeneration may a)
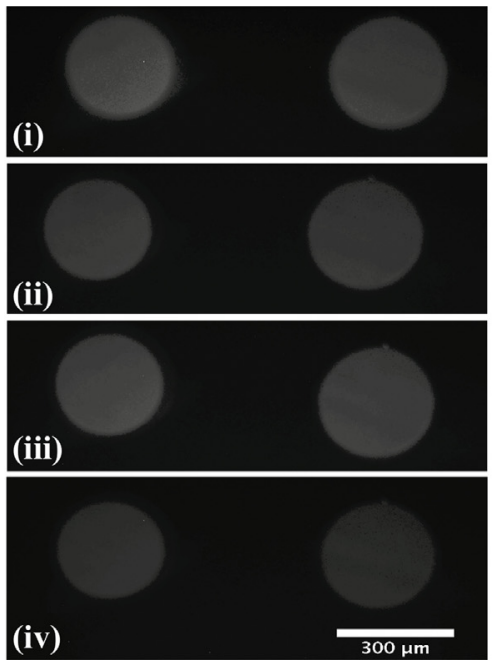

b)

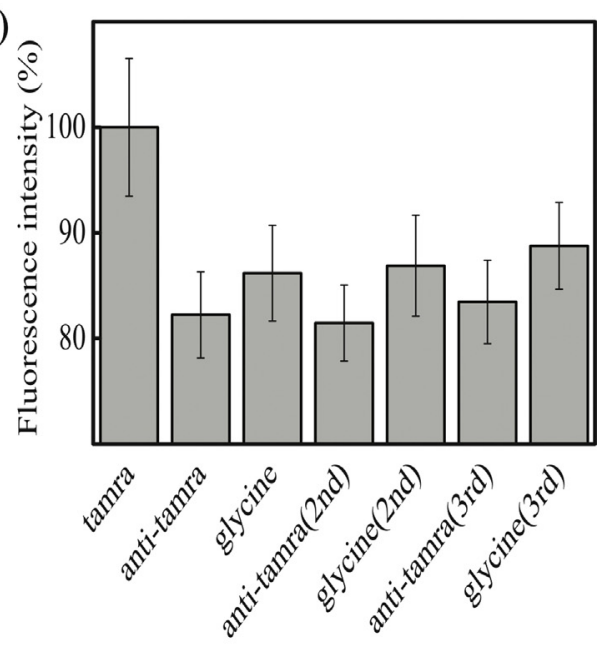

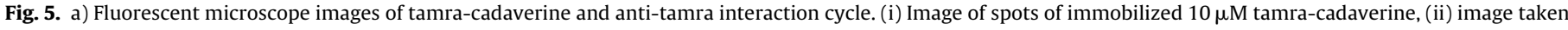

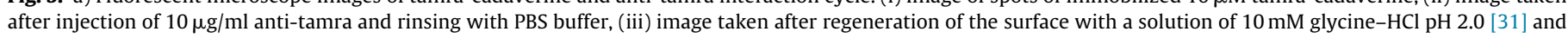
(iv) image taken after an additional injection of anti-tamra and rinsing with PBS buffer. b) Changes in fluorescence intensity during three reaction cycles. 
be explained by the low quenching efficiency after complex formation. Indeed, as characterized in the literature, quenching efficiency is not expected to exceed $\sim 57 \%$ of the initial intensity of the free tamra fluorophore diluted in a buffer solution [30]. In addition, since the average distance between tamra-cadaverine immobilized on the SU-8 surface was approximately $7 \mathrm{~nm}$ (deduced from Fig. 4c), not all immobilized tamra-cadaverine molecules can be involved in a complex with anti-tamra because of possible steric hindrance. Therefore, we quantified the intensity of fluorescence on each tamra-cadaverine spot and in each step of the association/regeneration cycle described in Fig. 5a. Fig. 5b summarizes the variation of fluorescence intensity (after correction for the background intensity signal) during three cycles of injection and regeneration. Considering the initial fluorescence intensity of the immobilized tamra-cadaverine spots to be $100 \%$, we observed a drop in the intensity of fluorescence to approximately $82 \%$ after anti-tamra/tamra-cadaverine complex formation on the SU-8 surface. The fluorescence signal was recovered up to $86 \%$ after regeneration with $10 \mathrm{mM}$ glycine- $\mathrm{HCl}, \mathrm{pH} 2.0$ solution. The loss of the signal from the immobilized tamra-cadaverine after regeneration might be due to desorption of tamra-cadaverine noncovalently bound to the surface but initially involved in complex formation and from undissociated complex that remains irreversibly on the surface. A second injection of anti-tamra again decreased the detected fluorescent signal to $81 \%$ and a second regeneration of the surface allowed total recovery of the signal intensity. A third cycle of association/regeneration showed similar variation of the fluorescence intensity demonstrating the specificity of the interaction on the pre-treated SU-8 surface.

\section{Conclusions}

We showed that UVO surface treatment is very promising compared to conventional wet or dry surface activation techniques known to significantly increase roughness [10]. We demonstrated that short exposure to UVO selectively oxidized SU-8 surfaces and increased hydrophilicity without damaging either thickness or surface roughness. Optimal conditions of hardbaking were established as being $120^{\circ} \mathrm{C}$ for $3 \mathrm{~h}$. Such UVO activated SU-8 surfaces were then used to covalent immobilization of tamra-cadaverine with high density. The binding process was specific and bound molecules were accessible for further antigen-antibody reactions. This specific and reversible reaction between molecules interacting with immobilized tamra-cadaverine molecules confirms that the SU-8 UVO activation method can be directly applied to the controlled immobilization of a range of (bio)molecules on SU-8 surfaces.

\section{Acknowledgment}

We would like to thank Camille Delezoide for insightful discussions which led to the development of the SU-8 UVO activation method described in the present paper; the characterization of Materials Platform of The Institute d'Alembert for the use of the FTIR instrument. This work has been supported by the DIM Nano'K Région Ile de France, the MIRODE project (ANR-11-BSV5-0017), the Laboratoire d'Excellence Nanosaclay and Palm for the project BOAB.

\section{References}

[1] A. del Campo, C. Greiner, SU-8: a photoresist for high-aspect-ratio and 3D submicron lithography, J. Micromech. Microeng. 17 (2007) R81-R95.

[2] P. Abgrall, V. Conedera, H. Camon, A.M. Gue, N.T. Nguyen, SU-8 as a structural material for labs-on-chips and microelectromechanical systems, Electrophoresis 28 (2007) 4539-4551.

[3] C. Delezoide, J. Lautru, J. Zyss, I. Ledoux-Rak, C.T. Nguyen, Vertically coupled polymer microresonators for optofluidic label-free biosensors, in: J.E. Broquin,
G.N. Conti (Eds.), Integrated Optics: Devices, Materials, and Technologies, Xvi, 2012.

[4] C. Delezoide, M. Salsac, J. Lautru, H. Leh, C. Nogues, J. Zyss, M. Buckle, I. LedouxRak, C.T. Nguyen, Vertically coupled polymer microracetrack resonators for label-free biochemical sensors, IEEE Photon. Technol. Lett. 24 (2012) 270-272.

[5] M. Nordstrom, R. Marie, M. Calleja, A. Boisen, Rendering SU-8 hydrophilic to facilitate use in micro channel fabrication, J. Micromech. Microeng. 14 (2004) 1614-1617.

[6] T. Sikanen, L. Heikkila, S. Tuornikoski, R.A. Ketola, R. Kostiainen, S. Franssila, T. Kotiaho, Performance of SU-8 microchips as separation devices and comparison with glass microchips, Anal. Chem. 79 (2007) 6255-6263.

[7] S.L. Tao, K.C. Popat, J.J. Norman, T.A. Desai, Surface modification of SU-8 for enhanced biofunctionality and nonfouling properties, Langmuir 24 (2008) 2631-2636.

[8] G. Blagoi, S. Keller, A. Johansson, A. Boisen, M. Dufva, Functionalization of SU-8 photoresist surfaces with IgG proteins, Appl. Surf. Sci. 255 (2008) 2896-2902.

[9] A. Deepu, V.V.R. Sai, S. Mukherji, Simple surface modification techniques for immobilization of biomolecules on SU-8, J. Mater. Sci. Mater. Med. 20 (2009) 25-28.

[10] M. Joshi, N. Kale, R. Lal, V.R. Rao, S. Mukherji, A novel dry method for surface modification of SU-8 for immobilization of biomolecules in Bio-MEMS, Biosens. Bioelectron. 22 (2007) 2429-2435.

[11] R. Marie, S. Schmid, A. Johansson, L.E. Ejsing, M. Nordstrom, D. Hafliger, C.B.V. Christensen, A. Boisen, M. Dufva, Immobilisation of DNA to polymerised SU-8 photoresist, Biosens. Bioelectron. 21 (2006) 1327-1332.

[12] V. Jokinen, P. Suvanto, S. Franssila, Oxygen and nitrogen plasma hydrophilization and hydrophobic recovery of polymers, Biomicrofluidics 6 (2012) $16501-16510$

[13] Y. Ji, K. Yuan, J.N. Chung, Numerical simulation of wall roughness on gaseous flow and heat transfer in a microchannel, Int. J. Heat Mass Transf. 49 (2006) 1329-1339.

[14] H.B. Winzeler, G. Belfort, Enhanced performance for pressure-driven membrane processes - the argument for fluid instabilites, J. Membr. Sci. 80 (1993) 35-47.

[15] J.S. Chang, S.C. Eom, G.Y. Sung, J.H. Shin, On-chip, planar integration of Er doped silicon-rich silicon nitride microdisk with SU-8 waveguide with sub-micron gap control, Opt. Express 17 (2009) 22918-22924.

[16] C. Cao, S.W. Birtwell, J. Hogberg, A. Wolff, H. Morgan, D.D. Bang, Surface modification of photoresist SU-8 for low autofluorescence and bioanalytical applications, in: 15th International Conference on Miniaturized Systems for Chemistry and Life Science, Seattle, Washington, USA, 2011.

[17] L.P. Haack, A.M. Straccia, J.W. Holubka, A. Bhurke, M. Xie, L.T. Drzal, Chemistry of surface modification with UV/ozone for improved intercoat adhesion in multilayered coating systems, Surf. Interface Anal. 29 (2000) 829-836.

[18] H.Y. Nie, M.J. Walzak, B. Berno, N.S. McIntyre, Atomic force microscopy study of polypropylene surfaces treated by UV and ozone exposure: modification of morphology and adhesion force, Appl. Surf. Sci. 144-145 (1999) 627-632.

[19] M.L. Sham, J. Li, P.C. Ma, J.K. Kim, Cleaning and functionalization of polymer surfaces and nanoscale carbon fillers by UV/Ozone treatment: a review (vol 43, pg 1537, 2009), J. Compos. Mater. 43 (2009) 3328

[20] M. Mrksich, G.M. Whitesides, Using self-assembled monolayers that present oligo(ethylene glycol) groups to control the interactions of proteins with surfaces, in: J.M. Harris, S. Zalipsky (Eds.), Poly(Ethylene Glycol): Chemistry and Biological Applications, 1997, pp. 361-373.

[21] C. Nogues, H. Leh, C.G. Langendorf, R.H.P. Law, A.M. Buckle, M. Buckle, Characterisation of peptide microarrays for studying antibody-antigen binding using surface plasmon resonance imagery, Plos One 5 (2010) e12152-e12158.

[22] C. Nogues, H. Leh, J. Lautru, O. Delelis, M. Buckle, Efficient antifouling surface for quantitative surface plasmon resonance based biosensor analysis, Plos One 7 (2012) e44287-e44297.

[23] Y. Sanogo, A.F. Obaton, C. Delezoide, J. Lautru, M. Lievre, J. Dubard, I. LedouxRak, C.T. Nguyen, Phase sensitive-optical low coherence interferometer: a new protocol to evaluate the performance of optical micro-resonators, J. Lightwave Technol. 31 (2013) 111-117.

[24] M.E. Fischer, Amine coupling through EDC/NHS: a practical approach, in: N.J. Mol, M.J.E. Fischer (Eds.), Surface Plasmon Resonance, Humana Press, 2010, pp. 55-73.

[25] S. Keller, G. Blagoi, M. Lillemose, D. Haefliger, A. Boisen, Processing of thin SU-8 films, J. Micromech. Microeng. 18 (2008) 125020-125030.

[26] Z.Y. Zhang, P. Zhao, G.Z. Xiao, B.R. Watts, C.Q. Xu, Sealing SU-8 microfluidic channels using PDMS, Biomicrofluidics 5 (2011) 046503-046508.

[27] C http://www.microchem.com

[28] C.J. Chang, C.S. Yang, L.H. Lan, P.C. Wang, F.G. Tseng, Fabrication of a SU-8-based polymer-enclosed channel with a penetrating UV/ozone-modified interior surface for electrokinetic separation of proteins, J. Micromech. Microeng. 20 (2010) $115031-115042$.

[29] Y.L. Wang, J.H. Pai, H.H. Lai, C.E. Sims, M. Bachman, G.P. Li, N.L. Allbritton, Surface graft polymerization of SU-8 for bio-MEMS applications, J. Micromech. Microeng. 17 (2007) 1371-1380.

[30] A.K. Chen, Z.L. Cheng, M.A. Behlke, A. Tsourkas, Assessing the sensitivity of commercially available fluorophores to the intracellular environment, Anal. Chem. 80 (2008) 7437-7444.

[31] N. Backmann, C. Zahnd, F. Huber, A. Bietsch, A. Pluckthun, H.P. Lang, H.J. Guntherodt, M. Hegner, C. Gerber, A label-free immunosensor array using single-chain antibody fragments, Proc. Natl. Acad. Sci. U.S.A. 102 (2005) 14587-14592. 